\title{
HORuS transmission spectroscopy of 55 Cnc e
}

\author{
H. M. Tabernero, ${ }^{1,2 \star C}$. Allende Prieto, ${ }^{3,4}$ M. R. Zapatero Osorio, ${ }^{2}$ \\ J. I. González Hernández, ${ }^{3,4}$ C. del Burgo, ${ }^{5,4}$ R. García López, ${ }^{3,4}$ R. Rebolo, ${ }^{3,4}$ \\ M. Abril-Abril, ${ }^{6}$ R. Barreto, ${ }^{3,4}$ J. Calvo Tovar, ${ }^{3,4}$ A. Díaz Torres, ${ }^{3,4}$ \\ P. Fernández Izquierdo, ${ }^{3,4}$ M.F. Gómez-Reñasco, ${ }^{3,4}$ F. Gracia-Témich, ${ }^{3,4}$ E. Joven, ${ }^{3,4}$ \\ J. Peñate Castro, ${ }^{3,4}$ S. Santana-Tschudi, ${ }^{3,4}$ F. Tenegi, ${ }^{3,4}$ and H. D. Viera Martín 6 \\ ${ }^{1}$ Instituto de Astrofísica e Ciências do Espaço, Universidade do Porto, CAUP, Rua das Estrelas, 4150-762 Porto, Portugal \\ ${ }^{2}$ Centro de Astrobiología (CSIC-INTA), Carretera de Ajalvir km 4, Torrejón de Ardoz, 28850 Madrid, Spain \\ ${ }^{3}$ Dpto. Astrofísica, Universidad de La Laguna, 38206 La Laguna, Tenerife, Spain \\ ${ }^{4}$ Instituto de Astrofísica Canarias, vía Láctea s/n, 38205 La Laguna, Tenerife, Spain \\ ${ }^{5}$ Instituto Nacional de Astrofísica, İĄ Óptica y Electrónica, Luis Enrique Erro 1, Sta. Ma. Tonantzintla, Puebla, Mexico \\ ${ }^{6}$ Gran Telescopio Canarias, Cuesta de San José, s/n, 38712, BreÃśa Baja, La Palma, Spain
}

Accepted: 2020 August 19; Received: 2020 January 6

\begin{abstract}
The High Optical Resolution Spectrograph (HORuS) is a new high-resolution echelle spectrograph available on the $10.4 \mathrm{~m}$ Gran Telescopio Canarias (GTC). We report on the first HORuS observations of a transit of the super-Earth planet 55 Cnc e. We investigate the presence of $\mathrm{Na}$ I and $\mathrm{H} \alpha$ in its transmission spectrum and explore the capabilities of HORuS for planetary transmission spectroscopy. Our methodology leads to residuals in the difference spectrum between the in-transit and out-of-transit spectra for the Na I doublet lines of $(3.4 \pm 0.4) \times 10^{-4}$, which sets an upper limit to the detection of line absorption from the planetary atmosphere that is one order of magnitude more stringent that those reported in the literature. We demonstrate that we are able to reach the photon-noise limit in the residual spectra using HORuS to a degree that we would be able to easily detect giant planets with larger atmospheres. In addition, we modelled the structure, chemistry and transmission spectrum of 55 Cnc e using state-of-the-art open source tools.
\end{abstract}

Key words: planets and satellites: atmospheres - planets and satellites: individual: $55 \mathrm{Cnc}$ e

\section{INTRODUCTION}

Planets orbiting stars are ubiquitous, as demonstrated by more than 4000 planets discovered to date ${ }^{1}$. With the pace of exoplanetary discoveries still increasing thanks to multiple transiting and radial velocity surveys carried out with e.g. space missions like Kepler, K2, CoRoT, and TESS (Ricker 2014); the ground-based spectrographs such as HARPS (Mayor et al. 2003), HARPS-N (Cosentino et al. 2012), HIRES (Vogt et al. 1994), CARMENES (Quirrenbach et al. 2016), and MARVELS (Alam et al. 2015); and new and future instruments like ESPRESSO (Pepe et al. 2010), CHEOPS (Rando et al. 2018), HPF (Mahadevan et al. 2014), JWST (Gardner et al. 2006), PLATO (Rauer et al. 2014), NIRPS (Wildi et al. 2017),

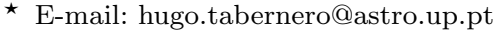

1 https://exoplanetarchive.ipac.caltech.edu
}

and SPIRou (Moutou et al. 2015). Exoplanetary research is now approaching the deep study and characterization of the atmospheres of the extrasolar planets.

Transiting exoplanets present a unique opportunity to characterize their atmospheres, and learn about their chemical composition. Charbonneau et al. (2002) reported the first detection of an exoplanet atmosphere by means of Hubble Space Telescope spectroscopy. These authors detected the resonance doublet of $\mathrm{NaI}$ in the Jupiter-size planet HD 209458 b. Casasayas-Barris et al. (2020) showed that this detection is due to the Rossiter-McLaughlin (RM) effect and not to the atmosphere of the planet. In spite of that result, Charbonneau et al. (2002) opened a new era in the study of exoplanets. The first detection of an exoplanet atmosphere with a ground-based telescope was made by Redfield et al. (2008), who identified the absorption of the sodium resonance lines caused by the 
atmosphere of HD 189733 b. Line absorption of $\mathrm{Na}, \mathrm{K}$, $\mathrm{He}, \mathrm{CO}, \mathrm{CH}_{4}$, or water vapour has already been reported in the atmospheres of tens of giant gaseous planets (e.g., Barman et al. 2015; Allart et al. 2017; Sheppard et al. 2017; Chen et al. 2018; Nortmann et al. 2018; Parmentier et al. 2018; Alonso-Floriano et al. 2019; Seidel et al. 2019). In addition, atomic absorption features due to Fe I,II and TiI,II have been detected in the atmosphere of the ultra-hot Jupiter KELT-9 b (Hoeijmakers et al. 2018, 2019). These measurements represent a step forward in our understanding of the chemistry of planetary atmospheres and our ability to constrain planet formation theories. The study of exoplanetary atmospheres is also moving towards the exploration of the atmospheres of rocky planets (i.e., super-Earths), but these atmospheres are harder to detect.

Spectroscopy is a powerful tool used to detect both broad and narrow features in the transmission spectra of transiting exoplanets, all of which are important ingredients to unveil their chemistry. The High Optical Resolution Spectrograph (HORuS) at the Gran Telescopio Canarias (GTC) offers an excellent opportunity for this task, given its moderate spectral resolution $(\mathrm{R} \approx 25,000)$ at optical wavelengths, and large collecting area, which is critical for achieving a high signal-to-noise ratio $(\mathrm{S} / \mathrm{N})$ during the limited time window of the planetary transit.

In this work, we analyse the HORuS commissioning data for a single transit of the hot and dense super-Earth planet $55 \mathrm{Cnc}$ e, which is orbiting one of the brightest planet-host stars known to have a super-Earth planet (Bourrier et al. 2018). The 55 Cnc system is an interesting multiplanetary system with five known planets that have been discovered during the last decades (see Butler et al. 1997; McArthur et al. 2004; Marcy et al. 2002; Fischer et al. 2008). 55 Cnc e is a supereath planet of $7.99 \mathrm{M}_{\oplus}$ and a planetary radius of $1.875 R_{\oplus}$. It orbits its parents star with a short period of $\approx 0.7 \mathrm{~d}$ at a distance to the host star $55 \mathrm{Cnc}$ of $0.01 \mathrm{au}$. This paper consists of the following sections: The HORuS observations and data reduction procedure are described in Sect. 2. The extraction of the planetary signal is documented in Sect. 3. In Sect. 4 we discuss our results and compare them to those obtained in previous works. Finally, in Sect. 5 we present the impact of our findings.

\section{OBSERVATIONS}

A series of spectroscopic observations covering one transit of the super-Earth $55 \mathrm{Cnc}$ e (see Table 1) was obtained with $\mathrm{HORuS}^{2}$, which is a moderate resolution echelle spectrograph $(\mathrm{R} \approx 25,000)$ in operation at the $10.4 \mathrm{~m}$ Gran Telescopio Canarias (GTC). It collects light at the Nasmyth-B focal plane, shared with OSIRIS, using a $3 \times 3$ integral field unit (IFU, $2.1 \times 2.1$ arcsec) with microlenses, into optical fibres that form a pseudo-slit at the spectrograph entrance, fitted with another set of microlenses.

2 http://research.iac.es/proyecto/abundancias/horus/ index.php
Table 1. Orbital and physical parameters for 55 Cnc and 55 Cnc e

\begin{tabular}{lcc}
\hline \hline Parameter & Value & Reference \\
\hline \hline & Stellar properties & \\
\hline$T_{\text {eff }}$ & $5353 \pm 62 \mathrm{~K}$ & Sousa et al. (2018) \\
$\log g$ & $4.30 \pm 0.14 \mathrm{dex}$ & Sousa et al. (2018) \\
{$[\mathrm{Fe} / \mathrm{H}]$} & $0.30 \pm 0.04 \mathrm{dex}$ & Sousa et al. (2018) \\
$\xi$ & $1.01 \pm 0.10 \mathrm{~km} \mathrm{~s}{ }^{-1}$ & Sousa et al. (2018) \\
$M_{*}$ & $0.97 \pm 0.09 \boldsymbol{M}_{\odot}$ & Bourrier et al. (2018) \\
$R_{*}$ & $1.73 \pm 0.04 R_{\odot}$ & Bourrier et al. (2018) \\
\hline & & \\
\hline & Planet properties & \\
$M_{p}$ & $0.7365474 \pm 0.0000014 \mathrm{~d}$ & Bourrier et al. (2018) \\
$R_{p}$ & $7.99 \pm 0.33 \boldsymbol{M}_{\oplus}$ & Bourrier et al. (2018) \\
$K_{1}$ & $1.875 \pm 0.05 R_{\oplus}$ & Bourrier et al. (2018) \\
$a$ & $6.02 \pm 0.24 \mathrm{~m} \mathrm{~s}^{-1}$ & Bourrier et al. (2018) \\
$\gamma$ & $0.01544 \pm 0.00005$ au & Bourrier et al. (2018) \\
$\boldsymbol{e}$ & $27.45145 \pm 0.00089 \mathrm{~km} \mathrm{~s}^{-1}$ & Bourrier et al. (2018) \\
$\omega$ & $0.05 \pm 0.03$ & Bourrier et al. (2018) \\
\hline
\end{tabular}

The light is dispersed with a $79 \mathrm{gr} \mathrm{mm}^{-1}$ echelle grating and cross-dispersed with three prisms, providing nearly continuous coverage between 3800 and $6900 \AA$ split over 27 spectral orders. The detector is a $4096 \times 409615-\mu m$-pixel Fairchild CCD.

We observed one transit of 55 Cnc e using HORuS at the GTC during the night of 12 Dec 2018. This night and the preceding one were part of a commissioning run for HORuS. The CCD was read with no binning, and a readout time of $90 \mathrm{~s}$. With a sampling of 6 pixels per resolution element in the spectral direction, and about the same number of pixels sampling each of the nine fibres in the IFU in the spatial direction, the 16-bit electronics can provide a maximum signal-to-noise ratio of several thousand per exposure. We took spectra of the Th-Ar hollow-cathode for wavelength calibration during the transit, with a cadence of approximately $1 \mathrm{~h}$. We obtained four $900 \mathrm{~s}$ exposures followed by twenty $450 \mathrm{~s}$ integrations, spanning slightly more than $4 \mathrm{~h}$, and including the transit. Each $450 \mathrm{~s}$ exposure provided a signal-to-noise per resolution element in excess of 1800 at $550 \mathrm{~nm}$, and about half of that at $420 \mathrm{~nm}$. The observations span over $\approx 4.2 \mathrm{~h}$, whereas the transit lasted for $\approx 1.6 \mathrm{~h}$. A total of 10 spectra were acquired during the planetary transit of $55 \mathrm{Cnc}$ e. In addition, we started observing at an airmass of 1.55 , whereas the observations finished at an airmass of 1.05 .

\section{DATA ANALYSIS}

\subsection{Data reduction}

The chain ${ }^{3}$, a custom-made data reduction pipeline for HORuS written in IDL, was used to process the data, performing bias removal, finding, tracing, and extraction of the aper-

\footnotetext{
3 https://github.com/callendeprieto/chain
} 
tures corresponding to the spectral orders. Wavelength calibration involved fitting third or -order polynomials to one to two dozen thorium lines in each echelle order, identified in an atlas made with the Tull spectrograph (Allende Prieto 2001), and interpolating linearly the wavelength solutions between the Th-Ar exposures preceding and following each stellar spectrum. The collapse of the orders along the slit was done by adding the counts along the spatial direction of the detector. By taking ratios of two consecutive out-oftransit spectra we find that the distribution of the residuals for the central regions of the orders in the red part of the spectrum is well described by a Gaussian with a $\sigma \simeq 0.0018$, which is equivalent to a signal-to-noise ratio per pixel of $\sqrt{2} / 0.0018 \simeq 800$, or 1900 per resolution element, confirming the expectations based on the Poisson noise $\left(\sqrt{N_{\text {counts }}}\right)$ for the accumulated stellar signal.

\subsection{Instrumental corrections}

Before attempting the extraction of the absorption signature imprinted by the planet's atmosphere in the spectrum of the star, we decided to analyse two particular spectral regions: the $\mathrm{Na}$ I doublet (5889.95 and $5895.92 \AA$ ) and the $\mathrm{H} \alpha$ line $(6562.80 \AA)$. HORuS is not stabilized thermally or mechanically, as it is the case of, e.g., ESPRESSO (Pepe et al. 2010), HARPS (Mayor et al. 2003), and CARMENES (Quirrenbach et al. 2016). Consequently, variations in the point spread function of the instrument, inducing changes of up to $10 \%$ in scales of hours, are typically found. We found that stability issues can be addressed by carefully aligning all the observed spectra by working in small wavelength regions of interest. Each individual spectroscopic observation has to be aligned with the others in terms of wave and flux. Spectral alignment is critical to extract the planetary signature, as the wavelength solution must be as precise as possible. To that aim, we generated an Fe I synthetic spectrum by means of turbospectrum ${ }^{4}$ (Plez 2012). In addition, we used a MARCS model atmosphere (Gustafsson et al. 2008) of $\mathrm{T}_{\text {eff }}=5250 \mathrm{~K}, \log g=4.5 \mathrm{dex}$, and $[\mathrm{M} / \mathrm{H}]=0.25 \mathrm{dex}^{5}$, alongside an FeI line list extracted from the Vienna Atomic Line Database (VALD3; Ryabchikova et al. 2015). We adopted iSpec (Blanco-Cuaresma et al. 2014) to cross-correlate each individual stellar observation with the generated Fe I synthetic spectrum. As a result we could measure the radial velocity corrections $\left(\sigma \approx 0.2 \mathrm{~km} \mathrm{~s}^{-1}\right.$, see Table A1) required to shift each individual spectrum to the rest frame.

Variations in the effective spectral resolution of HORuS also have to be taken into account to extract the planetary signal. Thus, we calculated a nominal resolution by measuring the width of Th lines in the calibration frames. The full width at half-maximum $(F W H M)$ of the cross-correlation given by iSpec of each individual Th-Ar exposure with itself (divided by $\sqrt{2}$ ) will give us an effective resolution for each exposure. At this point, we can homogenize all the observations to the exact same resolution, by means of convolving each spectrum with a Gaussian kernel that

4 https://github.com/bertrandplez/Turbospectrum2019

5 The closest MARCS model to the parameters given in Table 1

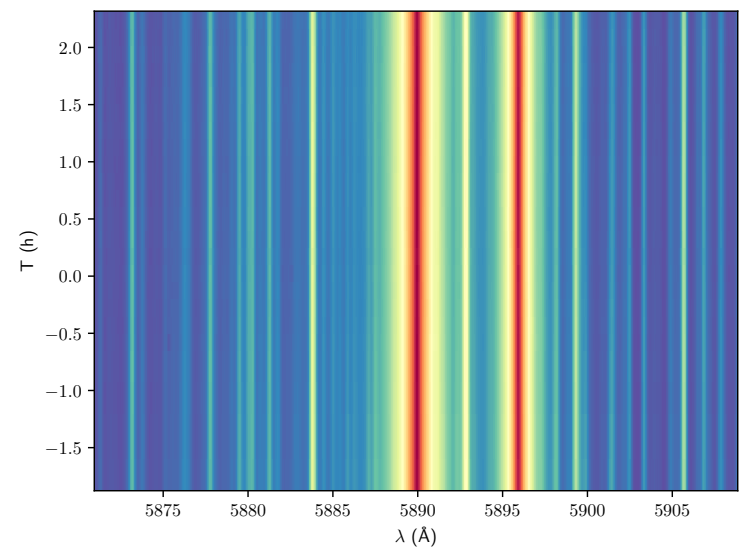

Figure 1. All HORuS spectra are shown vertically ordered in time (the earliest spectra are at the bottom). Time $0.0 \mathrm{~h}$ corresponds to the mid-transit according to the planetary ephemeris given in Table 1. The blue colour represents the continuum and all other colours stand for atomic absorption features (Na I doublet lines are the deepest). All stellar features are well aligned vertically and the flux continuum is nearly identical for all spectra.

accounts for the missing broadening.

\subsection{Extraction of the planetary signal}

The flux changes from exposure to exposure, due to variations in airmass and atmospheric transparency. Individual exposures must be corrected for these effects, and we used a low-order polynomial in order to have set of spectra in the same flux scale. The final result of flux alignment and wavelength correction is shown in Fig. 1. We separated the spectra into two categories, in-transit and out-of-transit spectra, by means of the ephemeris given by Bourrier et al. (2018). Then, we computed, wavelength by wavelength, the median of the out-of-transit spectra to generate a master spectrum. We employed the median to compute the out-of-transit spectrum because it is a non-skewed estimator more resilient to the influence of "bad pixels" (i.e. cosmic rays) in an individual exposure. We can divide each observation by this master spectrum to get a set of residual spectra that can be later combined to extract the signature of the planetary transit.

The telluric correction was done in an iterative manner, since the wavelength ranges of interest to this work contain heavily blended telluric lines to correct for (as well as the instrument resolution). However, it is possible to correct them without completely removing the planetary signature. Thus, we decided to employ the sysrem algorithm (Tamuz et al. 2005; Mazeh et al. 2007) to correct these lines. sysrem is an iterative detrending algorithm that is used to remove artefacts in any set of residual spectra (after diving by the master spectrum). This algorithm has been successfully employed in the literature (e.g., Alonso-Floriano et al. 2019; Hawker et al. 2018; Nugroho et al. 2017) to retrieve planetary signatures. We needed five sysrem iterations 
to remove the remaining telluric residuals and achieving the photon-noise limit. This gives us a set of residual spectra clean of any tentative remaining stellar and telluric residuals. We can combine the in-transit spectra after correcting them for the velocity of the planet derived using the ephemeris given by Bourrier et al. (2018) (see Table 1). After all the residual in-transit spectra are shifted to the planet rest frame we can merge them (using a median, in a wavelength by wavelength basis) to extract the final planetary signal.

It is important to mention that sysrem can partially remove the planet signal during each iteration. In consequence, we need to check that our method is not completely removing the planetary signal. Following Ridden-Harper et al. (2016) we injected a simulated planetary signal with an intensity of 0.005 to mimic the NaI lines. In Fig. 2 we show how our method does not remove any planetary signal, and that we can recover the injected model by means of only five sysrem iterations. We performed the analysis described above for both $\mathrm{Na}$ I doublet and the $\mathrm{H} \alpha$ line, we retrieve an absorption depth at about $(4.1 \pm 0.3) \times 10^{-3}$ for both $\mathrm{Na}$ I lines. Whereas for $\mathrm{H} \alpha$ we retrieve a similar value of $(4.4 \pm 0.7) \times 10^{-3}$. In consequence, these two retrieved values reveal that sysrem leaves the majority of the artificially injected planet signature intact.

\section{DISCUSSION}

The resulting planetary transmission spectrum centred at the resonance doublet of $\mathrm{Na}$ I and $\mathrm{H} \alpha$ is shown in Figs. 3 and A2. We have not detected $\mathrm{Na}$ I in $55 \mathrm{Cnc}$ e, as expected from its high mean molecular weight atmosphere contributing to only a small per cent of its planetary radius (Bourrier et al. 2018). However, we can infer an upper limit to the presence of these two lines. We have performed a bootstrap process to calculate the upper limit to the presence of the Na I and $\mathrm{H} \alpha$ lines. To that aim, we made groups of seven spectra to generate 120 transmission spectra in both spectral regions. Unfortunately, we have only a few observations and we had to make a compromise performing simulated spectra. Moreover, we need to allow variation to perform around one hundred simulations without affecting the statistics of each individual simulation. All in all, thanks to these simulations we can calculate the accuracy achievable using HORuS data.

Our calculations gave us an upper limit of $(3.4 \pm 0.4) \times 10^{-4}$ to the presence of the NaI doublet lines. This value represents a factor of $\approx 10$ improvement with respect to the previous measurements reported by Ridden-Harper et al. (2016). In addition, we have inferred an upper limit of $(7 \pm 1) \times 10^{-4}$ for the $\mathrm{H} \alpha$ line. The inferred upper limits are accurate to the first significant digit according to our bootstrap calculations. This is due to the relatively small number of statistically independent samples that can be drawn by means of the bootstrap method. The previous measurements in the Na I wavelength range were based on four transits of $55 \mathrm{Cnc}$ e observed with HARPS/ESO $3.6 \mathrm{~m}$ and HARPS-N/TNG. Despite the non-detection of $\mathrm{Na}$ in $55 \mathrm{Cnc}$ e, we have demonstrated that we are able to achieve the photon-noise limit of the exoplanetary data using HORuS to a degree that we will be able to detect giant planets. These planets have larger atmospheres and transmission depths above $10^{-3}$ (at the $3 \sigma$ level) that can be easily detected, as indicated by our results.

In addition, our upper limit on $\mathrm{H} \alpha$ is in good agreement with the conclusions of Ehrenreich et al. (2012), as they do not detect any hints of neutral hydrogen in the transmission spectrum of $55 \mathrm{Cnc}$ e. However, Tsiaras et al. (2016) detected two tentative HCN features in the infrared wavelength range (at 1.42 and $1.54 \mu \mathrm{m}$ ) suggesting a lightweight atmosphere.

Our estimated upper limit to the depth of the Na I doublet lines and $\mathrm{H} \alpha$ needs to be compared to the predictions of theoretical models. To that aim we computed an atmospheric structure using the HELIOS ${ }^{6}$ code (Malik et al. 2017, 2019, see Figs. A3 and A4). HELIOS allowed us to generate tailored models taking into account the irradiation of the parent star. HELIOS requires fundamental parameters for both the star and the planet, as well as a chemical composition. Estimates for those parameters were taken from Bourrier et al. (2018), and we assumed solar abundances from (Asplund et al. 2009). The HELIOS model was used in FastChem ${ }^{7}$ (Stock et al. 2018) to compute the number densities for several atomic and molecular species in the atmosphere of the planet (see Fig. A5). In addition, we calculated the transmission spectrum of 55 Cnc e thanks to the petitRADTRANS ${ }^{8}$ code (Mollière et al. 2019) alongside the HELIOS model and the particle number densities we have calculated with FastChem. We used the high-resolution option of petitRADTRANS to compute two synthetic spectra of $55 \mathrm{Cnc}$ e using two different $\mathrm{C} / \mathrm{O}$ ratios (solar and twice solar composition). The modelling of the planetary atmosphere is a necessary step to know what are the limits of HORuS. We plot these synthetic models in Fig. A6. Further inspection of our synthetic modelling reveals that the depths of the modelled sodium lines are $\approx 3 \times 10^{-5}$ with respect to the continuum. Therefore, we need to improve the noise level at least by a factor of 30 to be able to confidently detect any $\mathrm{NaI}$ in $55 \mathrm{Cnc}$ e. Regarding $\mathrm{H} \alpha$, our models are consistent with a non-detection of this feature at the resolution of HORuS independently of the $\mathrm{C} / \mathrm{O}$ ratio (Fig. A6). Thus, ruling out atmospheres with lower mean molecular weight than those of our models requires a better signalto-noise ratio. In consequence, future observations with an improved signal-to-noise ratio are required in order to be able to confidently detect ( $3 \sigma$ detection) the atmosphere of 55 Cnc e.

\section{CONCLUSIONS}

In this work, we have analysed the transit of the super-Earth $55 \mathrm{Cnc}$ e in the Na I doublet region to explore the capabilities of HORuS as a high-resolution echelle spectrograph. The main conclusions of this work can be summarized as follows:

\footnotetext{
6 https://github.com/exoclime/HELIOS

7 https://github.com/exoclime/FastChem

8 https://petitradtrans.readthedocs.io/en/latest/
} 

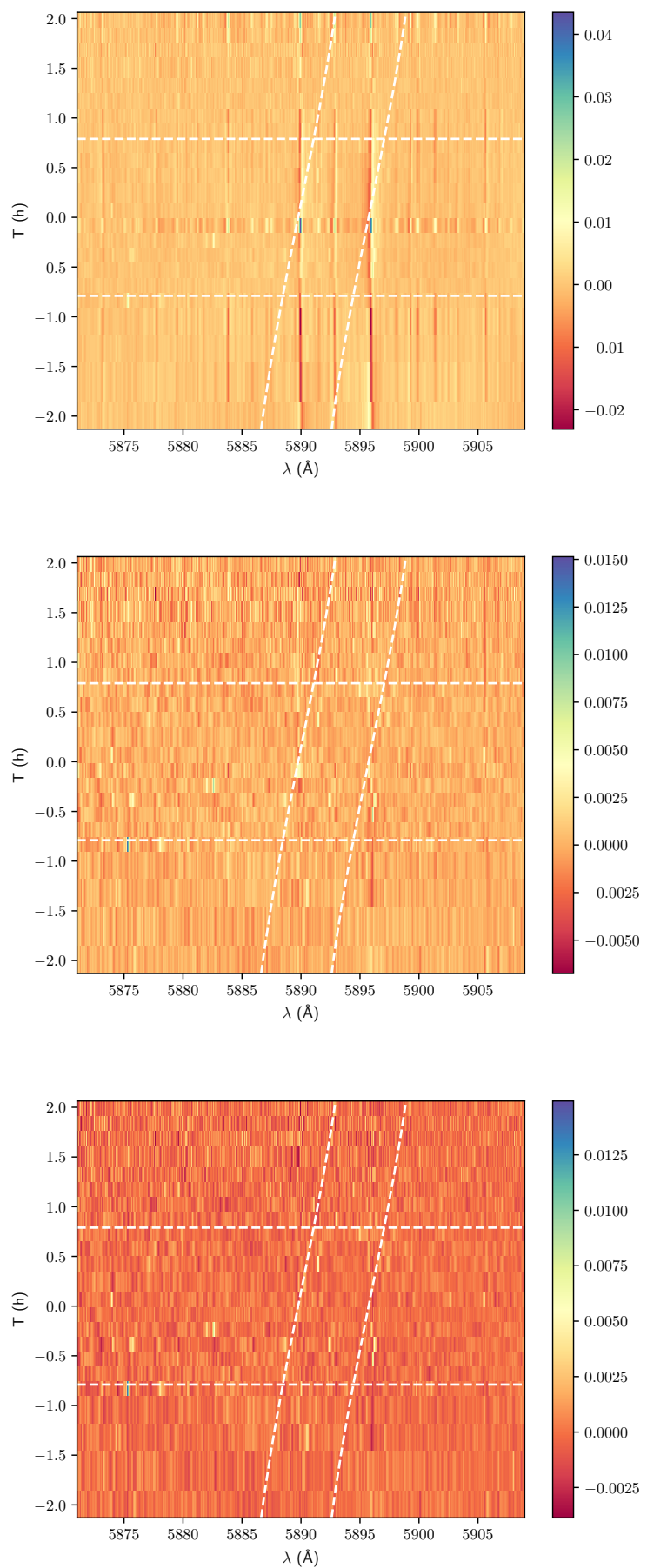
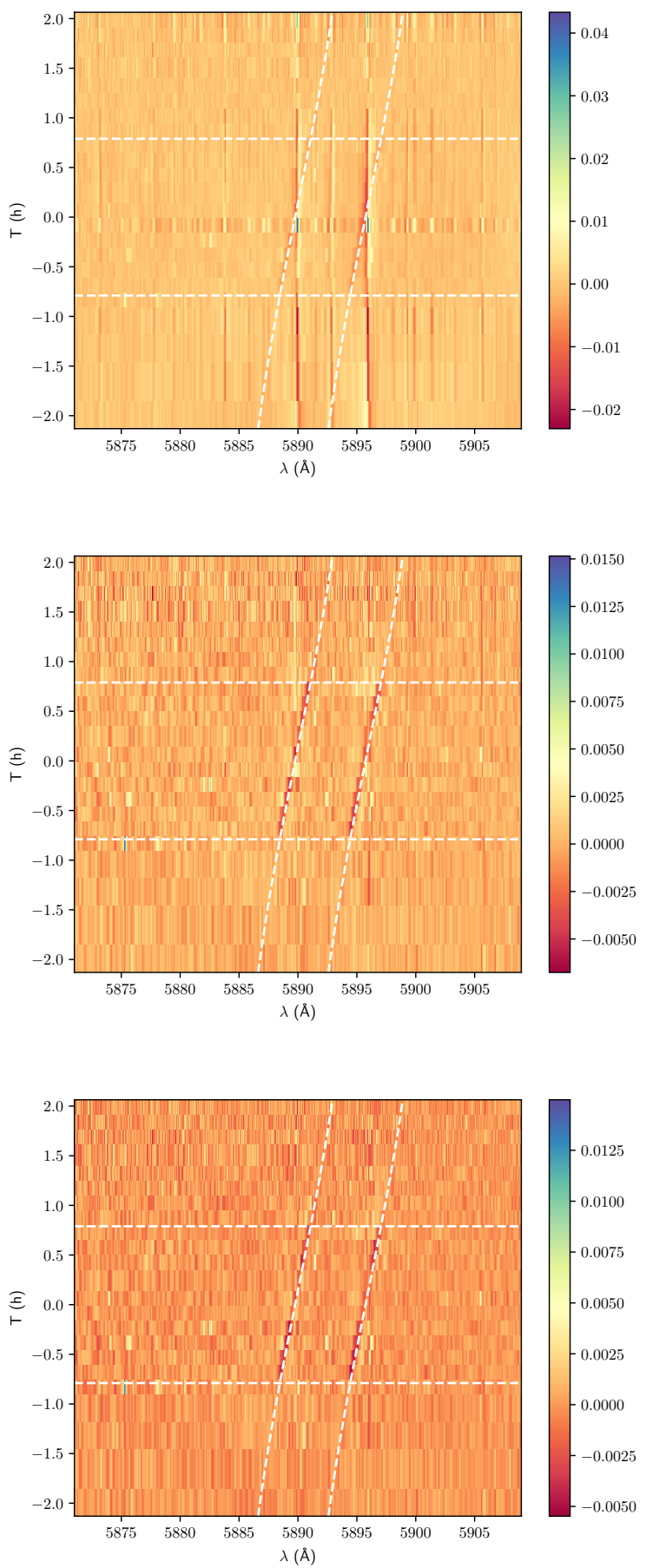

Figure 2. Visual representation of our sysrem implementation applied to the Na I doublet. The left-hand column represents the HORuS data, whereas the right-hand column shows the same data with an injected planetary signal. Each row represents different steps of our sysrem implementation. Here we represent our results for the removal of the master spectrum (top panels), as well as three and five sysrem iterations. The transit window is given by the two horizontal dashed lines, whereas the planet trace is drawn by the two vertical curves.

(i) We estimated uncertainties in the derived radial velocities at about $0.2 \mathrm{~km} \mathrm{~s}^{-1}$. This is typical for instruments like HORuS, which have not been stabilized thermally, optically, or mechanically.

(ii) We confirm that the high sampling of the signal pro- vided by HORuS, with order widths of about 60 pixels in the spatial direction and 6 pixels in the spectral direction, can provide a signal-to-noise ratio of about 1900 per resolution element in a single 450-s exposures for 55 Cnc.

(iii) Our methodology has allowed us to place a limit on 


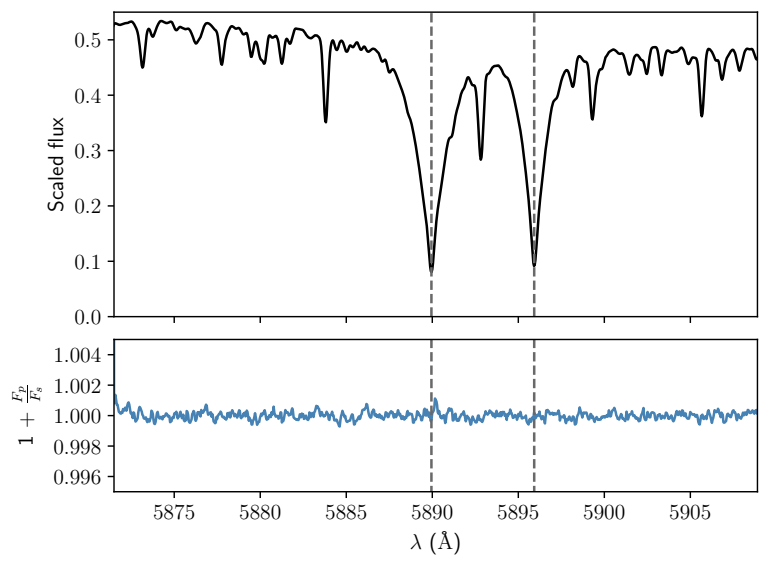

Figure 3. Final master stellar spectrum (top) and final transmission spectrum (bottom) for $55 \mathrm{Cnc}$ e in the $\mathrm{NaI}$ region. The sodium doublet wavelengths are marked by the two vertical dashed lines.

the detection of the planetary signal in the by NaI resonance doublet of $(3.4 \pm 0.4) \times 10^{-4}$, improving by a factor of approximately 10 a previous limit given by Ridden-Harper et al. (2016).

(iv) We have also studied the noise level for the $\mathrm{H} \alpha$ line, proving an limit to the presence of $\mathrm{H} \alpha:(7 \pm 1) \times 10^{-4}$.

(v) Our modelling indicated that the signal to noise of our observations has to be increased by a factor of 30 to detect the atmosphere of 55 Cnc e at the $3 \sigma$ level.

(vi) We have demonstrated that we are able to reach the photon-noise limit of the exoplanetary data using HORuS to a degree that we can detect planets with larger atmospheres (i.e., giant planets). These planets will be easily detected, as indicated by the results of this study.

\section{ACKNOWLEDGEMENTS}

We would to thank the anonymous referee for the insightful comments and suggestions that improved the manuscript of the paper. This work was supported by Fundação para a Ciência e a Tecnologia (FCT) through the research grants UID/FIS/04434/2019, UIDB/04434/2020 and UIDP $/ 04434 / 2020$. HMT and MRZO acknowledge financial support from the Spanish Ministerio de Ciencia, Innovación y Universidades through projects AYA2016-79425C3-2. HMT also acknowledges the FCT - Fundação para a Ciência e a Tecnologia through national funds (PTDC/FISAST/28953/2017) and by FEDER - Fundo Europeu de Desenvolvimento Regional through COMPETE2020 - Programa Operacional Competitividade e Internacionalização (POCI-01-0145-FEDER-028953). JIGH acknowledges financial support from the Spanish Ministry of Science, Innovation and Universities (MICIU) under the 2013 Ramón y Cajal program RYC-2013-14875. CAP, JIGH, and RR acknowledge financial support from the Spanish Ministry project MICIU AYA2017-86389-P. CdB acknowledges the funding of his sabbatical position through the Mexican national council for science and technology (CONACYT grant CVU No.
448248). CdB is also thankful for the support from the Jesus Serra Foundation Guest Program.

\section{DATA AVAILABILITY}

The data underlying this article will be shared on reasonable request to the corresponding author.

\section{REFERENCES}

Alam S., et al., 2015, ApJS, 219, 12

Allart R., Lovis C., Pino L., Wyttenbach A., Ehrenreich D., Pepe F., 2017, Astronomy and Astrophysics, 606, A144

Allende Prieto C., 2001, arXiv e-prints, pp astro-ph/0111172

Alonso-Floriano F. J., et al., 2019, A\&A, 621, A74

Asplund M., Grevesse N., Sauval A. J., Scott P., 2009, ARA\&A, 47,481

Barman T. S., Konopacky Q. M., Macintosh B., Marois C., 2015, ApJ, 804, 61

Blanco-Cuaresma S., Soubiran C., Heiter U., Jofré P., 2014, A\&A, 569, A111

Bourrier V., et al., 2018, Astronomy and Astrophysics, 619, A1

Butler R. P., Marcy G. W., Williams E., Hauser H., Shirts P., 1997, ApJ, 474, L115

Casasayas-Barris N., et al., 2020, A\&A, 635, A206

Charbonneau D., Brown T. M., Noyes R. W., Gilliland R. L., 2002, The Astrophysical Journal, 568, 377

Chen G., et al., 2018, A\&A, 616, A145

Cosentino R., et al., 2012, Harps-N: the new planet hunter at TNG. p. $84461 \mathrm{~V}$, doi:10.1117/12.925738

Ehrenreich D., et al., 2012, A\&A, 547, A18

Fischer D. A., et al., 2008, ApJ, 675, 790

Gardner J. P., et al., 2006, Space Sci. Rev., 123, 485

Gustafsson B., Edvardsson B., Eriksson K., Jørgensen U. G., Nordlund Å., Plez B., 2008, A\&A, 486, 951

Hawker G. A., Madhusudhan N., Cabot S. H. C., Gandhi S., 2018, ApJ, 863, L11

Hoeijmakers H. J., et al., 2018, Nature, 560, 453

Hoeijmakers H. J., et al., 2019, Astronomy and Astrophysics, 627, A165

Mahadevan S., et al., 2014, in Ramsay S. K., McLean I. S., Takami H., eds, Vol. 9147, Ground-based and Airborne Instrumentation for Astronomy V. SPIE, pp $543-$ 552, doi:10.1117/12.2056417, https://doi.org/10.1117/12. 2056417

Malik M., et al., 2017, AJ, 153, 56

Malik M., Kitzmann D., Mendonça J. M., Grimm S. L., Marleau G.-D., Linder E. F., Tsai S.-M., Heng K., 2019, AJ, 157, 170

Marcy G. W., Butler R. P., Fischer D. A., Laughlin G., Vogt S. S., Henry G. W., Pourbaix D., 2002, ApJ, 581, 1375

Mayor M., et al., 2003, The Messenger, 114, 20

Mazeh T., Tamuz O., Zucker S., 2007, in Afonso C., Weldrake D., Henning T., eds, Astronomical Society of the Pacific Conference Series Vol. 366, Transiting Extrapolar Planets Workshop. p. 119 (arXiv: astro-ph/0612418)

McArthur B. E., et al., 2004, ApJ, 614, L81

Mollière P., Wardenier J. P., van Boekel R., Henning T., Molaverdikhani K., Snellen I. A. G., 2019, A\&A, 627, A67

Moutou C., Boisse I., Hébrard G., Hébrard E., Donati J. F., Delfosse X., Kouach D., 2015, in SF2A-2015: Proceedings of the Annual meeting of the French Society of Astronomy and Astrophysics. pp 205-212 (arXiv:1510.01368)

Nortmann L., et al., 2018, Science, 362, 1388

Nugroho S. K., Kawahara H., Masuda K., Hirano T., Kotani T., Tajitsu A., 2017, AJ, 154, 221 


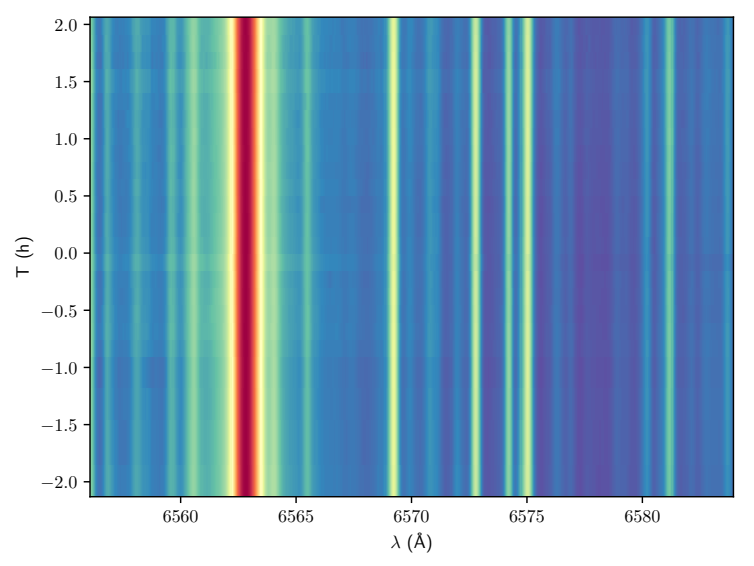

Figure A1. Same as Fig. 1 but for $\mathrm{H} \alpha$

Parmentier V., et al., 2018, A\&A, 617, A110

Pepe F. A., et al., 2010, in Ground-based and Airborne Instrumentation for Astronomy III. p. $77350 \mathrm{~F}$, doi: $10.1117 / 12.857122$

Plez B., 2012, Turbospectrum: Code for spectral synthesis, Astrophysics Source Code Library (ascl:1205.004)

Quirrenbach A., et al., 2016, in Ground-based and Airborne Instrumentation for Astronomy VI. p. 990812, doi:10.1117/12.2231880

Rando N., et al., 2018, in Proc. SPIE. p. 106980K, doi: $10.1117 / 12.2311300$

Rauer H., et al., 2014, Experimental Astronomy, 38, 249

Redfield S., Endl M., Cochran W. D., Koesterke L., 2008, The Astrophysical Journal, 673, L87

Ricker G. R., 2014, Journal of the American Association of Variable Star Observers (JAAVSO), 42, 234

Ridden-Harper A. R., et al., 2016, A\&A, 593, A129

Ryabchikova T., Piskunov N., Kurucz R. L., Stempels H. C., Heiter U., Pakhomov Y., Barklem P. S., 2015, Phys. Scr., 90, 054005

Seidel J. V., et al., 2019, A\&A, 623, A166

Sheppard K. B., Mandell A. M., Tamburo P., Gand hi S., Pinhas A., Madhusudhan N., Deming D., 2017, ApJ, 850, L32

Sousa S. G., et al., 2018, A\&A, 620, A58

Stock J. W., Kitzmann D., Patzer A. B. C., Sedlmayr E., 2018, MNRAS, 479, 865

Tamuz O., Mazeh T., Zucker S., 2005, MNRAS, 356, 1466

Tsiaras A., et al., 2016, ApJ, 820, 99

Vogt S. S., et al., 1994, HIRES: the high-resolution echelle spectrometer on the Keck 10-m Telescope. p. 362, doi:10.1117/12.176725

Wildi F., et al., 2017, in Shaklan S., ed., Vol. 10400, Techniques and Instrumentation for Detection of Exoplanets VIII. SPIE, pp 321 - 335, doi:10.1117/12.2275660, https://doi.org/10. $1117 / 12.2275660$

\section{APPENDIX A: EXTRA MATERIAL}

This paper has been typeset from a $\mathrm{TE}_{\mathrm{E}} \mathrm{X} / \mathrm{LATEX}$ file prepared by the author.

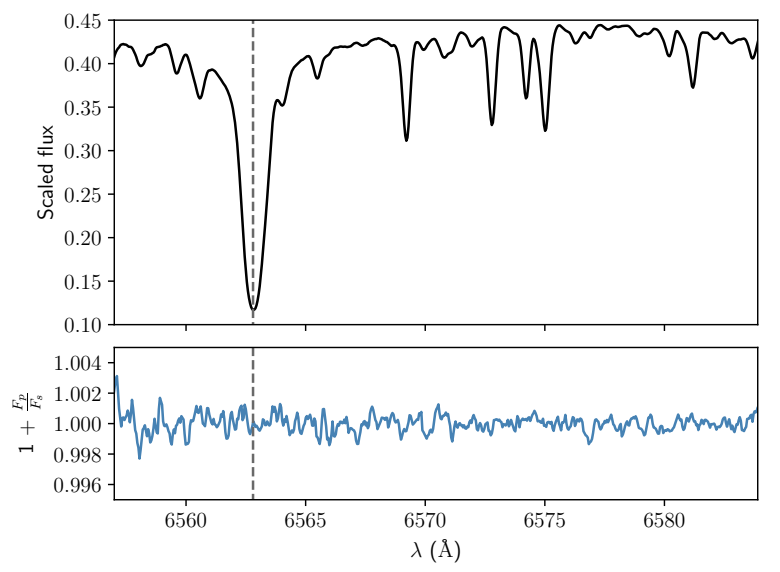

Figure A2. Final master stellar spectrum (top) and final transmission spectrum (bottom) for $55 \mathrm{Cnc}$ e in the $\mathrm{H} \alpha$ region. The $\mathrm{H} \alpha$ wavelength is denoted by the vertical dashed line.

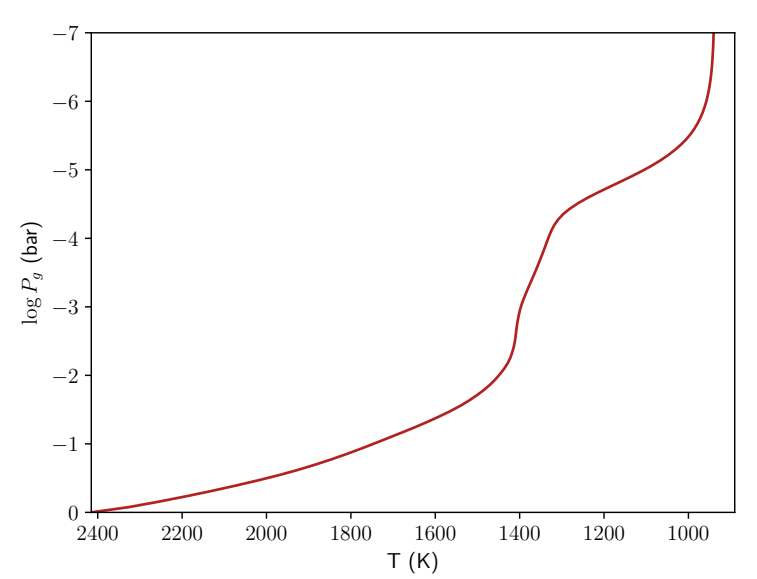

Figure A3. 55 Cnc e atmospheric P-T structure generated with the HELIOS code (Malik et al. 2017, 2019). 
Table A1. Observational time series for 55 Cnc. We provide Modified Julian Dates (MJD), radial velocities (RVs) for the Na I and $\mathrm{H} \alpha$ wavelengths as well as the effective resolution (in terms of $F W H M$ ) and the width of the convolution kernel $\left(F W H M_{\mathrm{k}}\right)$ for each individual exposure.

\begin{tabular}{lccccccc}
\hline \hline mjd & $\begin{array}{c}\mathrm{t}_{\mathrm{exp}} \\
(\mathrm{s})\end{array}$ & $\begin{array}{c}\mathrm{RV}(\mathrm{NaI}) \\
\left(\mathrm{km} \mathrm{s}^{-1}\right)\end{array}$ & $\begin{array}{c}F W H M(\mathrm{NaI}) \\
\left(\mathrm{km} \mathrm{s}^{-1}\right)\end{array}$ & $\begin{array}{c}F W H M_{\mathrm{k}}(\mathrm{NaI}) \\
\left(\mathrm{km} \mathrm{s}^{-1}\right)\end{array}$ & $\begin{array}{c}\mathrm{RV}(\mathrm{H} \boldsymbol{\alpha}) \\
\left(\mathrm{km} \mathrm{s}^{-1}\right)\end{array}$ & $\begin{array}{c}F W H M(\mathrm{H} \alpha) \\
\left(\mathrm{km} \mathrm{s}^{-1}\right)\end{array}$ & $\begin{array}{c}F W H M_{\mathrm{k}}(\mathrm{H} \alpha) \\
\left(\mathrm{km} \mathrm{s}^{-1}\right)\end{array}$ \\
\hline 58465.01122 & 900 & $27.79 \pm 0.25$ & $13.46 \pm 0.14$ & 5.12 & $27.72 \pm 0.21$ & $14.93 \pm 0.15$ & 4.43 \\
58465.02281 & 900 & $27.53 \pm 0.24$ & $13.38 \pm 0.13$ & 5.32 & $27.57 \pm 0.20$ & $14.75 \pm 0.15$ & 5.02 \\
58465.03939 & 900 & $27.40 \pm 0.23$ & $13.14 \pm 0.13$ & 5.89 & $27.50 \pm 0.19$ & $14.50 \pm 0.15$ & 5.7 \\
58465.05083 & 900 & $27.22 \pm 0.23$ & $13.02 \pm 0.13$ & 6.15 & $27.43 \pm 0.18$ & $14.37 \pm 0.14$ & 6.02 \\
58465.06241 & 450 & $27.28 \pm 0.23$ & $12.90 \pm 0.13$ & 6.40 & $27.51 \pm 0.18$ & $14.23 \pm 0.14$ & 6.34 \\
58465.06864 & 450 & $27.29 \pm 0.23$ & $12.83 \pm 0.13$ & 6.54 & $27.44 \pm 0.18$ & $14.16 \pm 0.14$ & 6.50 \\
58465.07487 & 450 & $27.41 \pm 0.23$ & $12.76 \pm 0.13$ & 6.67 & $27.51 \pm 0.18$ & $14.09 \pm 0.14$ & 6.65 \\
58465.08109 & 450 & $27.60 \pm 0.23$ & $12.70 \pm 0.13$ & 6.79 & $27.71 \pm 0.18$ & $14.01 \pm 0.14$ & 6.82 \\
58465.08732 & 450 & $27.72 \pm 0.22$ & $12.63 \pm 0.13$ & 6.92 & $27.85 \pm 0.18$ & $13.94 \pm 0.14$ & 6.96 \\
58465.09354 & 450 & $27.91 \pm 0.24$ & $12.57 \pm 0.13$ & 7.02 & $27.73 \pm 0.19$ & $13.86 \pm 0.14$ & 7.16 \\
58465.09975 & 450 & $27.93 \pm 0.22$ & $12.50 \pm 0.13$ & 7.15 & $28.00 \pm 0.17$ & $13.80 \pm 0.14$ & 7.23 \\
58465.10598 & 450 & $28.01 \pm 0.22$ & $12.43 \pm 0.12$ & 7.27 & $28.16 \pm 0.17$ & $13.79 \pm 0.14$ & 7.25 \\
58465.11480 & 450 & $28.10 \pm 0.21$ & $12.45 \pm 0.12$ & 7.23 & $28.23 \pm 0.17$ & $13.71 \pm 0.14$ & 7.40 \\
58465.12101 & 450 & $28.14 \pm 0.22$ & $12.69 \pm 0.13$ & 6.81 & $28.16 \pm 0.17$ & $13.96 \pm 0.14$ & 6.91 \\
58465.12724 & 450 & $28.20 \pm 0.22$ & $12.94 \pm 0.13$ & 6.32 & $28.27 \pm 0.17$ & $14.19 \pm 0.14$ & 6.43 \\
58465.13347 & 450 & $28.14 \pm 0.23$ & $13.19 \pm 0.13$ & 5.78 & $28.17 \pm 0.18$ & $14.43 \pm 0.14$ & 5.87 \\
58465.13969 & 450 & $28.05 \pm 0.23$ & $13.43 \pm 0.13$ & 5.20 & $28.10 \pm 0.18$ & $14.66 \pm 0.15$ & 5.27 \\
58465.14591 & 450 & $28.07 \pm 0.24$ & $13.68 \pm 0.14$ & 4.50 & $28.05 \pm 0.19$ & $14.90 \pm 0.15$ & 4.55 \\
58465.15214 & 450 & $28.13 \pm 0.25$ & $13.93 \pm 0.14$ & 3.65 & $28.13 \pm 0.19$ & $15.13 \pm 0.15$ & 3.72 \\
58465.15836 & 450 & $28.25 \pm 0.25$ & $14.17 \pm 0.14$ & 2.56 & $28.19 \pm 0.20$ & $15.37 \pm 0.15$ & 2.55 \\
58465.16730 & 450 & $28.44 \pm 0.26$ & $14.40 \pm 0.14$ & 0.00 & $28.41 \pm 0.20$ & $15.58 \pm 0.16$ & 0.00 \\
58465.17353 & 450 & $28.43 \pm 0.27$ & $14.30 \pm 0.14$ & 1.69 & $28.36 \pm 0.20$ & $15.48 \pm 0.16$ & 1.76 \\
58465.17975 & 450 & $28.30 \pm 0.27$ & $14.20 \pm 0.14$ & 2.39 & $28.24 \pm 0.21$ & $15.38 \pm 0.15$ & 2.49 \\
58465.18598 & 450 & $28.15 \pm 0.27$ & $14.10 \pm 0.14$ & 2.92 & $28.00 \pm 0.21$ & $15.28 \pm 0.15$ & 3.04 \\
\hline
\end{tabular}

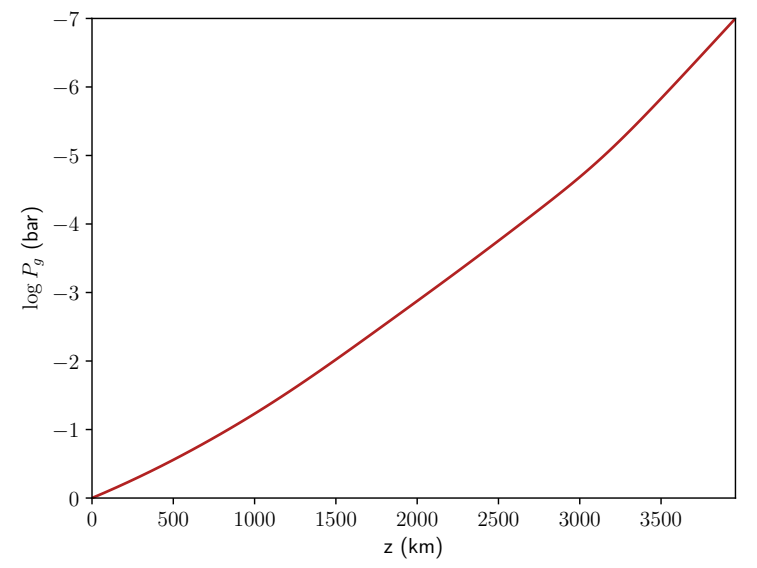

Figure A4. Same as Fig. A3 but for gaseous pressure as function of altitude $(\mathrm{z})$

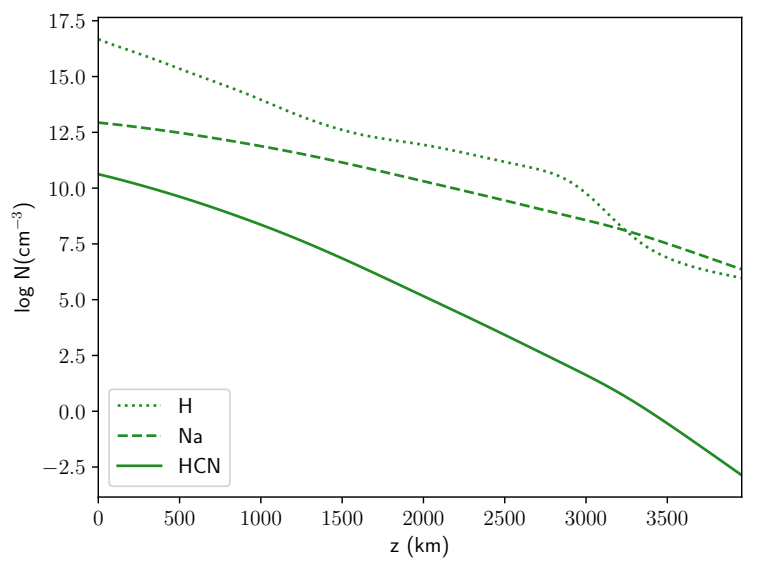

Figure A5. Number densities of $\mathrm{H}, \mathrm{HCN}$, and Na calculated with Fastchem (Stock et al. 2018) vs the model altitude ( $z$ ). These number densities correspond to a model with a solar $\mathrm{C} / \mathrm{O}$ ratio. 

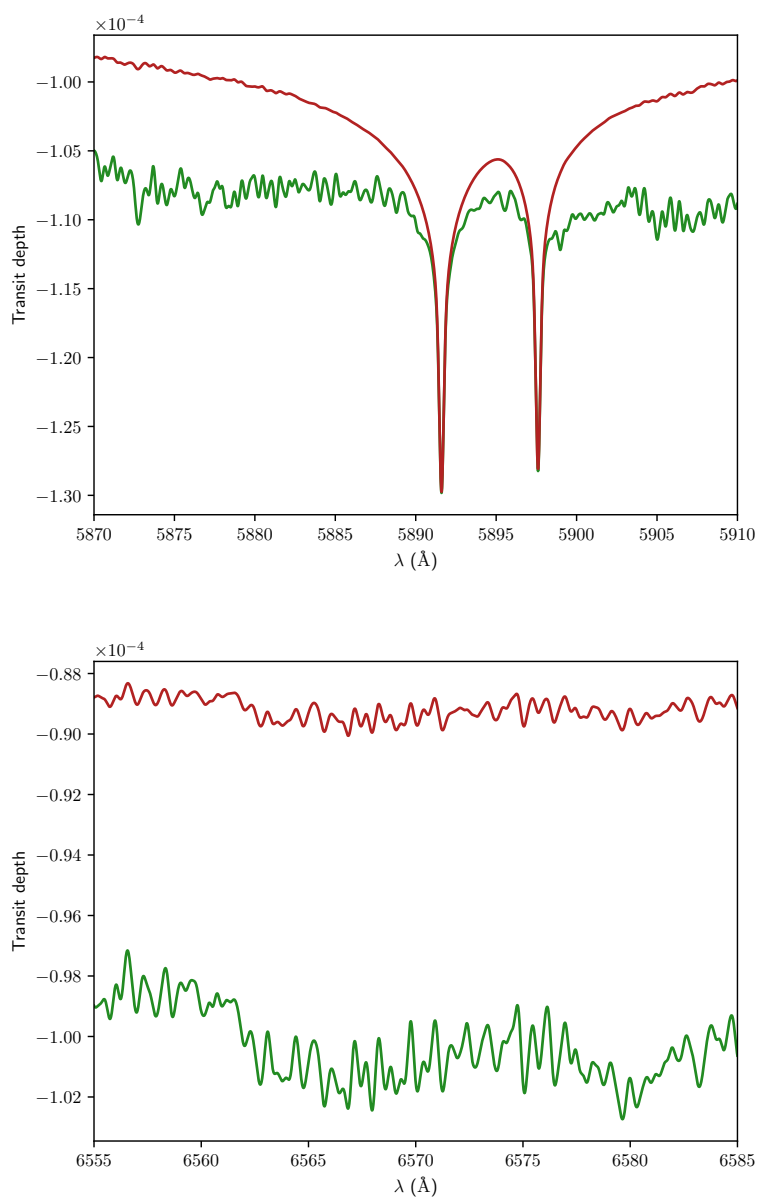

Figure A6. Our modelled synthetic spectra around Na I (top) and $\mathrm{H} \alpha$ (bottom) using petitRADTRANS for the optical range. The green line corresponds to a model with a solar $\mathrm{C} / \mathrm{O}$ ratio, whereas the red line represents twice the solar ratio. Both synthetic models have been degraded to the HORuS resolution as provided in Table A1 